\title{
Designing a Wellness Self-Management Tool for Older Adults - Results from a Field Trial of YourWellness
}

\author{
Julie Doyle, Lorcan Walsh, Antonella Sassu, Teresa McDonagh, \\ CASALA, Dundalk Institute of Technology, \\ Dublin Rd., Dundalk, Ireland \\ \{first.last@casala.ie\}
}

\begin{abstract}
It is recognized that empowering individuals to manage their own health and wellbeing will result in more cost-effective healthcare systems, improved health outcomes and will encourage healthy individuals to remain that way. With the advent of the quantifiedself movement in recent years, there has been an increase in technology applications supporting wellness self-management. Such applications allow people to self-track and self-report, with many providing feedback. However, little research in this area has examined how best to support older adults in health selfmanagement. This paper reports findings from a 5-month home deployment of YourWellness - an application that supports older adults in self-reporting on their wellbeing and provides feedback to promote positive wellbeing management. Our findings contribute to a greater understanding of older adults' attitudes and behaviours in relation to wellbeing self-management that can facilitate the creation of new, personalized health and wellbeing interventions for this population.
\end{abstract}

\section{Keywords}

Older adults; wellness; self-management; feedback

\section{INTRODUCTION}

Countries globally have been experiencing an unprecedented increase in the number of older adults. From 2006 to 2026, within the 65 and over age group, the dependency ratio ${ }^{1}$ is expected to increase from $16.4 \%$ to $25.1 \%$ for Ireland, and increase from $25.2 \%$ to $36.6 \%$ for the EU 25 [4]. In the US, the dependency ratio was $22 \%$ in 2010 and is estimated to climb to $35 \%$ in 2030 [27]. Older adults are a diverse group and many enjoy long-term, good health. However, to varying degrees, national health and social care systems are preparing for potentially higher incidences of chronic disease, such as diabetes, arthritis and COPD (chronic obstructive pulmonary disease) and resulting dependency problems. For example, the incidence of diabetes is set to double between 2005 and 2030 to an estimated number of 366 million people living with diabetes in 2030 [29]. There is a cost element. Across developed countries approximately half of healthcare spending is attributed to older adults. In the UK, hospital readmission has risen by over $30 \%$ due to lack of appropriate support at home [26]. There is thus a paradigm shift towards moving from the current, reactive model of healthcare to one that is more proactive and preventative. A critical challenge thus exists in supporting older adults to manage their wellbeing within their home environment.

This shift towards preventative health care has resulted in a growing interest in home-based assisted living technologies, designed for and with older adults [6], [14]. These include a broad

\footnotetext{
${ }^{1}$ The old dependency ratio is the ratio of those over 65 over the working population (15-64 years old).
}

spectrum: from remote health monitoring, electronic sensors and equipment such as fall detectors and bed alerts, to information and communication technologies (ICT) and environmental control systems (ECS). There has been a surge in the design of technology-based wellness applications that support people in actively monitoring their wellbeing, largely due to the wide availability of smart phones embedded with powerful sensors [15], [20]. Such applications promote wellbeing by providing the individual with some level of feedback based on collected data. However, there are issues with the majority of the technologies described above. Often they are not designed with older adults in mind, and critically, any data collected is typically sent to carers or clinicians rather than to the older adult themselves. Thus, little is known about how older adults might self-manage their own wellbeing and what might motivate them to do so.

This paper describes YourWellness, an iPad application designed to support older adults in self-reporting on their wellbeing and to provide feedback on such, in terms of showing an overview of an individual's wellbeing, trends of their data over time and information and education on how to maintain or return to a healthy state of being. The overall aim of the application is to provide a holistic approach to wellbeing self-management. While incidences of chronic illness are high in older adults, and while monitoring of physiological symptoms is important, what is often ignored are the associated health issues, such as effects on mood, social interactions, physical activity or sleep.

In this paper, we present findings from a 5-month field trial of YourWellness with older adults. The purpose of this trial was to examine long-term adherence to self-reporting in an older population, to gauge opinions on whether the application increased awareness of one's wellbeing, whether it encouraged people to alter their behaviour in some way and whether people found the application, particularly the feedback provided, useful and usable. For this particular trial, following from a focus group discussion on parameters of wellness that older adults find important and are interested in managing [8], we focused on 3 areas of wellbeing, mood, sleep and social interactions. However, the application and its management system as described in the paper, support management of any area of wellbeing of interest to the person. Since this trial, we have added categories of falls prevention, nutrition, physical activity, blood pressure and weight management, with the user provided with a 'menu' of wellness parameters from which they can choose which categories they are interested in.

We found that older adults are interested in self-managing their wellness, they want to be more aware of their health and they are motivated to use such applications provided they are useful to them. Other results around how best to design wellness selfmanagement applications for this population are presented. We consider our main contribution to be an increased understanding of older adults' attitudes and behaviours in relation to wellbeing self-management and factors that affect acceptance and adoption 
of such, that can facilitate the creation of new, personalized health and wellbeing interventions for this population. Specifically, we found that older adults found line graphs to be intuitive and useful representations of their data and used these to dig down into their data and self-reflect; answering questions, in addition to receiving feedback, increased self-awareness; however ensuring these questions are not too repetitive, combined with maintaining fresh and engaging educational feedback/advice is necessary to encourage long-term adherence to wellness self-management. We also found that supporting the provision of context is important to allow people to make sense of anomalies in their data.

\section{Background and Related Work}

Recent times have seen the advent of the quantified-self movement, whereby individuals have begun to use technology devices to 'self-track'. Self-tracking typically involves recording some aspect of your life, including your work, sleep, exercise, diet or mood and supports self-management of such through the provision of feedback. This feedback is typically persuasive and aims to help the person to change their behaviour to live the lifestyle they wish to lead [10]. Some of these 'lifestyle' management applications include UbiFit [3], BeWell [15] and Fish ' $n$ ' Steps [19] and have been designed for general populations, not necessarily older adults. Li has examined how such technologies can support self-reflection and the types of questions people ask themselves while reflecting [18]. Such questions involved current status, history, goals, discrepancies, context and factors (long-term behavioural factors). Selfmanagement applications have also begun to appear in the health and wellness space. Monarca is a persuasive monitoring and feedback system for mental illness and is an excellent example of a closed feedback loop to patients $[1,20]$. There are also a number of noteworthy commercial applications, including Fitbit and the Nike+ Sportband.

The majority of health management applications focus on management of chronic illnesses, such as COPD, chronic heart failure, diabetes or blood pressure management [11, 21]. These support self-tracking, typically with wearable sensors such as a pulse oximeter or blood pressure cuff. They provide a limited form of feedback to the user (e.g. today's blood pressure value), but the goal is typically to provide feedback to a clinician to support remote management of health.

There is also much research in the space of health applications targeted specifically at older people including those to support functional ability [16] physical [7, 29], social [9] or cognitive wellbeing [12]. While some of these systems provide feedback to support health improvement, their long-term usage in real environments is limited. Furthermore, they tend to focus on one aspect of wellbeing, rather than providing a holistic approach as outlined in [26]. Lee and Dey [16] describe a sensorised system to monitor pill taking and phone usage in older populations, the aim of which is to increase participant awareness of their habits and anomalies in their habits (such as forgetting to take a pill or misdialing phone numbers). The authors highlight design

Permission to make digital or hard copies of all or part of this work for personal or classroom use is granted without fee provided that copies are not made or distributed for profit or commercial advantage and that copies bear this notice and the full citation on the first page. To copy otherwise, or republish, to post on servers or to redistribute to lists, requires prior specific permission and/or a fee.

Conference'10, Month 1-2, 2010, City, State, Country.

Copyright 2010 ACM 1-58113-000-0/00/0010 ...\$15.00. recommendations for such systems, including highlighting anomalies and supporting the retrieval of context. However, their work is based on case studies from just two participants both of whom interacted with visualisations of their recorded data only once.

\section{The YourWellness Framework}

This section describes the YourWellness framework, consisting of an end-user mobile application, a Web-based management application and a cloud-based service supporting communication. The framework supports the collection of data, feeds results back to end users and delivers educational content and advice.

\subsection{YourWellness Mobile Application}

For the purpose of this trial, participants self-reported on their mood, sleep and social interactions. Additional categories of wellness have since been integrated and the application framework is flexible, supporting the addition of any further areas of wellbeing that may be important to a particular individual. YourWellness has been fully co-designed with older adults. We have conducted interviews, focus groups, workshops and usability testing and received feedback from older adults on attitudes towards wellness management, preferred methods for inputting information, preferred visualisations of feedback and the type of content that would be required for such an application. We also ran workshops with clinicians to ensure the application's validity as a wellness tool. Details on our design process are described elsewhere [8]. Information gathered during this process was used to design the first prototype, as described below.

Each day, users of YourWellness answer 8-10 questions about their wellbeing and receive real-time feedback. At the highest level, a feedback 'wheel' provides a quick-glance overview of wellbeing (Fig. 1a). The wheel is divided into segments, based on the parameters of wellness that are being monitored and uses the traffic light metaphor as a high level indicator of wellbeing status. Thus, the interior part of the segment is coloured green if the individual is self-reporting that they are feeling well; orange if they are scoring relatively low; and red if they score very low. In collaboration with clinical specialists and taking into account existing guidelines such as the NHS NICE guidelines [23], we have developed a scoring algorithm that calculates a wellness score for determining whether a green, orange or red alert should be provided.

Clicking a particular segment of the feedback wheel brings the user to a graph of their self-report data to date (Fig. 1b). Educational/advice content is also provided as a series of messages delivered daily (Fig. 1c). These messages are targeted towards the user's current wellbeing state. For example, if a user is scoring in the orange zone for sleep they will get a message with a tip informing them how to improve their sleep hygiene. Such content has been defined for each type of alert in each category of wellbeing (i.e. sleep, mood and social), in collaboration with clinicians and by examining existing guidelines, for example from the $\mathrm{NHS}^{2}$ and the Harvard Sleep Centre $^{3}$. Examples of such message are shown in Table 1. In the social category, we suggest local classes and events that the person might be interested in. The overall aim of such feedback is to promote awareness of wellbeing and support improvement. While the goal of our research is to support self-management of

\footnotetext{
${ }^{2}$ http://guidance.nice.org.uk/PH16

${ }^{3} \mathrm{http}: / /$ healthysleep.med.harvard.edu/
} 
wellbeing in an older population, the YourWellness framework also supports sharing of data, thus it could potentially be used in various contexts, as explained in the next section.

Table 1. Examples of Advice/Educational Messages

\begin{tabular}{|l|l|}
\hline Sleep & $\begin{array}{l}\text { Avoid caffeine, alcohol, nicotine and other chemicals that } \\
\text { interfere with sleep for 4-6 hours before bedtime }\end{array}$ \\
\hline Sleep & $\begin{array}{l}\text { It may help to limit your bedroom activities to sleep. Keeping } \\
\text { computers, TVs and work materials out of the room will } \\
\text { strengthen the mental association between your bedroom and } \\
\text { sleep }\end{array}$ \\
\hline Sleep & $\begin{array}{l}\text { Light reading before bed is a good way to prepare yourself for } \\
\text { sleep }\end{array}$ \\
\hline Mood & $\begin{array}{l}\text { Dehydration makes a person tired and cranky. Sense of thirst } \\
\text { decreases with age, even for healthy older people. Therefore it } \\
\text { is important to drink water before you feel thirsty }\end{array}$ \\
\hline Mood & $\begin{array}{l}\text { Learning a new skill or achieving a goal, however minor, boosts } \\
\text { self-esteem and motivation }\end{array}$ \\
\hline Mood & $\begin{array}{l}\text { Research has shown that if you go for daily 10-minute walks, } \\
\text { overall energy levels and mood will be lifted within 3 weeks }\end{array}$ \\
\hline Social & $\begin{array}{l}\text { You might be interested in a 6-week course of tai chi for older } \\
\text { adults that is beginning next week in your local community } \\
\text { centre. The details are.... }\end{array}$ \\
\hline Social & $\begin{array}{l}\text { Did you know that the local swimming pool offers free passes } \\
\text { to senior citizens every Tuesday? }\end{array}$ \\
\hline
\end{tabular}

\subsection{YourWellness Management System}

Alongside the mobile user application, a web-based application has been developed to support its management. This system is cloud-based and provides a framework for easy management of the application. Key features include: the ability to add question sets related to any area of wellbeing and push these to the user; the ability to define how each question is weighted to generate an overall score for each category; the ability to add multimedia educational content (such as videos, interactive images and articles) related to an area of wellbeing and push these to the user; the ability to manage the frequency of question delivery to the user; the ability to manage and sort user responses, provide an overview of their state of wellbeing, their adherence to answering questions etc.

While this tool has initially been setup to support the research trial, it has potential to be used by clinicians and caregivers. For example, geriatric consultants who we have consulted have said that they would use such an application in conjunction with tele- health equipment as part of early discharge from hospital, to monitor not only patients' physiological vital signs, but also how they are feeling post-discharge. The management system supports the addition of any question set specific to an individual and feedback can be defined. The management system thus makes it easy for clinicians or carers to set up the user application for that user's specific needs.

\section{Home Deployment Study Design}

At time of writing, YourWellness has been deployed for a total of 7 months. However, the evaluation described in this paper was conducted at the end of month 5 of the deployment. The purpose of the evaluation was to (1) Determine older adults' opinions on the usefulness of the application, particularly the feedback; (2) Examine motivations to use such an application; (3) Examine the usability of the application and how easily older adults could interpret their data; (4) Examine whether it increased awareness of wellbeing.

\subsection{Participants and Procedure}

We recruited 8 older adult participants to take part in the study $(5 \mathrm{M}$ and $3 \mathrm{~F})$. However, one of the female participants withdrew due to illness. Participant demographics are shown in Table 1. Our exclusion criteria were those under the age of 60 and those who couldn't provide informed consent. Of the 7 participants who took part, 4 had their own iPad and 3 had never used an iPad before. Of these 3, 2 had previously used laptops or desktops to some extent (p59, p66). None of the 7 participants had previously used any health related application or used any form of technology for health purposes. However, 2 of the participants recorded their blood pressure in notebooks (p65, p69) while one recorded his blood sugars (p65).

We visited participants in their home to install the application, provide a tutorial on it and to carry out pre-trial interviews. We informed participants that they would have a new set of questions to answer daily, but that they should answer the questions whenever they liked, as we were interested in real-world usage rather than forced daily usage. We asked participants to self-report honestly and highlighted that their responses were not being sent to anyone other than themselves. We also informed participants that the study period was 2 months, but that they would have the opportunity to continue with the trial, using the application, for an ongoing period if they chose. We called participants after the initial 2-month period to ask if they were interested in continuing with the trial and all but one (p60) continued. Reasons for this are explained in the Adherence subsection of the results.

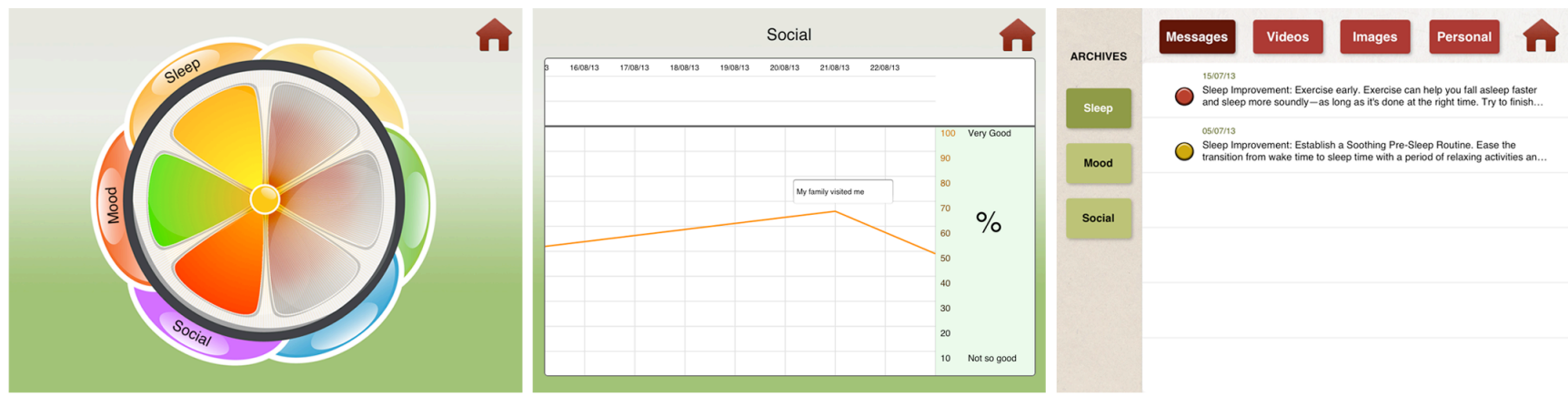

Figure 1. (a) Overview feedback wheel (b) Graph data for a person's self-reported quality of social interactions (c) Examples of educational advice messages for sleep 


\subsection{Data Captured}

We collected the following data for evaluation purposes: (1) Adherence to self-reporting. For this study, participants selfreported on 3 areas of wellbeing - mood, sleep and social interactions and also answered a daily trivia question. Mood questions were asked each day and social and sleep on alternative days. (2) Responses to questions. From this we calculated whether participants were in the green, orange or red zone for each wellbeing parameter measured as well as changes between these zones throughout the trial; (3) Post-trial interviews with participants. Data collected was qualitative. Transcription of responses yielded data for content analysis. Two coders identified important themes based on the frequency and intensity of participant responses; (4) Usability, measured during the interview through (a) Think-aloud protocol whereby the participant was asked to complete a number of tasks on the application and researchers observed their interactions and recorded their speech and (b) System Usability Questionnaire [2]; (5) Motivation to use and continue using such an application, measured through the Intrinsic Motivation Inventory (IMI) [5]. Intrinsic motivation is defined as an end-user's propensity to engage in activities of interest and the resultant promotion in learning and expansion of the individual's capacities [25]. We examined 4 subscales of the IMI - Interest/Enjoyment, Perceived Competence, Pressure/Tension and Value/Usefulness. This was administered during the post-trial interviews. Our findings are outlined in the following section.

\section{Results}

Table 2 shows participant demographics as well as scores for usability (SUS) and motivation to continue using (IMI subscales Int/Enj, Perc Comp, Pres/Tens and Val/Use). Before we discuss these results, we examine adherence.

Table 2. Participant demographic information and results from the SUS (where 100 is highest score) and IMI (where 7 is highest score, except for Pres/Tens scale where 1 is best score)

\begin{tabular}{|l|l|l|l|l|l|l|l|l|}
\hline P ID & G & Age & $\begin{array}{l}\text { Own } \\
\text { iPad }\end{array}$ & SUS & $\begin{array}{l}\text { Int / } \\
\text { Enj }\end{array}$ & $\begin{array}{l}\text { Perc } \\
\text { Comp }\end{array}$ & $\begin{array}{l}\text { Pres / } \\
\text { Tens }\end{array}$ & $\begin{array}{l}\text { Val / } \\
\text { Use }\end{array}$ \\
\hline 59 & $\mathrm{M}$ & 67 & $\mathrm{~N}$ & 90 & 4.7 & 6.8 & 1 & 6.25 \\
\hline 60 & $\mathrm{~F}$ & 69 & $\mathrm{~N}$ & 75 & 5 & 6 & 1 & 6.75 \\
\hline 64 & $\mathrm{M}$ & 77 & $\mathrm{Y}$ & 60 & 5.2 & 5.4 & 1.4 & 5 \\
\hline 65 & $\mathrm{M}$ & 75 & $\mathrm{Y}$ & 90 & 6 & 7 & 3.4 & 7 \\
\hline 66 & $\mathrm{M}$ & 68 & $\mathrm{~N}$ & 84 & 4.8 & 7 & 1 & 6.5 \\
\hline 67 & $\mathrm{~F}$ & 65 & $\mathrm{Y}$ & 93 & 7 & 7 & 1 & 7 \\
\hline 69 & $\mathrm{M}$ & 73 & $\mathrm{Y}$ & 93 & 5.5 & 6.4 & 2.6 & 7 \\
\hline Avg & & 70.6 & & 83.6 & 5.5 & 6.5 & 1.6 & 6.5 \\
\hline
\end{tabular}

\subsection{Adherence}

We collected data on adherence to self-reporting. The graph in Figure 2 indicates compliance to answering questions (i.e. selfreporting), calculated as the number of days in each month that questions were asked over the number of days in each month that questions were answered. As expected, adherence to selfreporting was highest during the first month, with an average of $79.2 \%$ adherence across participants. This dropped to $63.1 \%$ in month 2. After month 2, participant 60 dropped out of the trial as she felt that the categories of wellness were not relevant for her.
She felt managing nutrition and physical activities would be of more benefit for her personally. Since the trial, we have included other parameters of wellness and can now provide users with a choice of categories. We have included data from p60 here as she took part in the post-trial evaluations. Otherwise, all participants wanted to continue using the application past the initial 2-month study period. A technical issue with regard to participant 69 occurred during month 3 whereby this participant's iPad went into 'recovery mode' which effectively wiped his iPad and all its data. During the evaluation stage, the application was therefore evaluated with mock-data to prompt discussion. Participant 69 has since asked for the application to be loaded onto his iPad again so that he can continue using it.

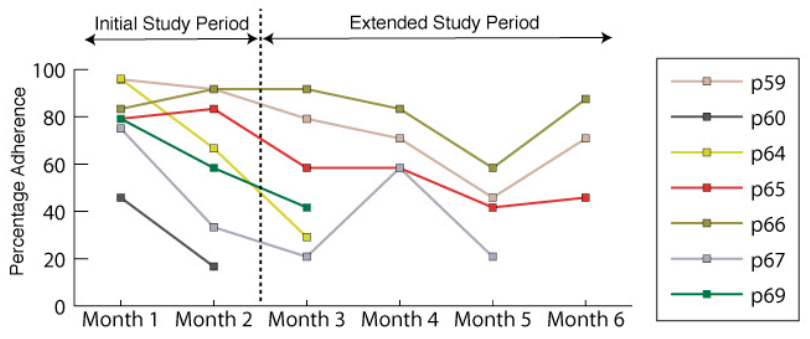

Figure 2. Adherence data throughout the trial

During month 5 , there was a period of 10 days when the server hosting the application was offline due to technical difficulties, which accounts for the sharp drop in self-reporting for this month. The end of month 5 also marked the end of the trial, when interviews were carried out with participants. So we can see that 3 participants have continued to use the application following the official end of the trial and at time of writing, participant 69 has also started to use the application again. Thus, while there were technical issues that impacted on the trial, this is the nature of longitudinal studies and such issues must be catered for.

\subsection{Usability}

The average usability score for each participant as measured by the SUS is shown in Table 2. The highest possible score on this scale is 100 . The average score across all participants is 83.6 , with a range from 60 to 93 indicating that participants found the application usable. During the post-trial usability test where we employed the think-aloud method we observed all participants were easily able to use and navigate the application. We asked participants to complete tasks that would require them to answer questions on the application, browse and navigate, look at their overview data (the feedback wheel), their graphs and their advice messages.

Only two participants (59 and 65) understood the concept of the traffic light metaphor - i.e. the use of green to indicate positive wellbeing, orange to indicate medium etc. All participants understood the graph and the advice messages and found this information very useful. This will be examined further in the Discussion section.

\subsection{Motivation}

\subsubsection{Interest and Enjoyment}

Interest and enjoyment are the most integral factors of intrinsic motivation. The mean interest and enjoyment score was 5.5 (standard deviation 0.8), where 7 is the highest possible score. In terms of the individual subscales used to assess motivation, interest and enjoyment had the lowest score. This was somewhat 
expected as it is unlikely that participants would 'enjoy' using a wellness management application. This was reflected by participant responses when completing the IMI whereby participants reported finding the application of interest, not 'fun' or 'enjoyable'.

\subsubsection{Perceived Competence}

Perceived competence within the IMI relates to an individual's perception of how capable they are at performing a certain task. In our study, this refers to the older adult's perception of how well they can use the YourWellness application. The mean perceived competence was 6.5 out of 7 (standard deviation 0.6). The high scores in perceived competence were reflected in the post-trial interviews and application walk-through, whereby we observed no issues with user interaction.

\subsubsection{Pressure/Tension}

The pressure/tension subscale assesses the level of tension a person feels while performing a certain task and is a negative predictor of intrinsic motivation. We felt it important to assess this for YourWellness as answering potentially sensitive questions on health and wellbeing might be stressful for some individuals. Furthermore, becoming more aware of one's wellbeing may also cause tension, particularly if that person is not doing so well. The mean score for pressure/tension was 1.6 (standard deviation 0.97), where a score of 1 is a positive score, indicating that users felt quite relaxed while using YourWellness.

\subsubsection{Value/Usefulness}

A person's perception of the value or usefulness of using an application such as YourWellness can act as a significant motivator for them to not only initially adopt it, but also to continue using it. The mean value/usefulness score was 6.5 out of 7 (standard deviation 0.7), indicating that our participants would find it useful to continue using YourWellness in the future. This scale on the IMI also asked participants (1) why they found the application useful and (2) why they think the application is important. Some responses to (1) included "making you more aware of your wellbeing", "learning things about yourself that you normally take for granted", "keeping an eye on your health", and "helping you take control of your health". With regard to (2) participants felt that such an application was important because it can "help you to understand how you're doing" and "improve your life".

\subsection{Other Findings}

We asked participants to name 3 good and 3 not so good things about the application. Positive aspects related to ease of use (7 participants), the usefulness of the feedback (5), the inclusion of relevant questions (2) and the inclusion of mood as a wellbeing parameter (2). With regard to negative aspects, most responses related to the repetitiveness of questions (5), the inclusion of parameters of wellbeing that the individual wasn't necessarily interested in (3), and the lack of facility to 'go back' and change your answer when answering questions (2).

\section{Discussion}

\subsection{Understanding Feedback}

During the post-trial interview, all participants commented on how essential feedback on wellbeing is to using an application like YourWellness. During interviews we asked participants to explain to us their understanding of the various feedback elements in the application. We began with the feedback wheel to gain an insight into whether participants had understood the use of green, orange and red in the interface. We found that the use of the traffic light metaphor was only understood by two participants, p59 and p65, despite this being highlighted during the deployment and initial training of the application. "You're doing the traffic light thing there, no?" (p59). We assume this lack of understanding was present throughout the trial. Some participants associated colours with the different parameters of wellbeing: "Is red sleep and yellow mood? No, sleep is yellow and red is social", (p67) while others thought they were just part of a 'fancy' interface or that they reflect 'your personality' - "Would they relate to your personality or something? Well, usually nice bright colours are associated with lively people, who want to be involved in things" (p60). This lack of understanding or interpretation didn't appear to have any negative effect on participants' desire or ability to use the application - participants weren't confused or weren't striving to understand what the colours might mean during the trial. As highlighted below, participants used the graphs to interpret and understand their own data, rather than the feedback wheel.

Following the study, to facilitate interpretation we have integrated an information box that appears when the feedback wheel is launched, providing a textual explanation along the lines of "Did you know that this petal can be green, orange or red? Green means...." While we don't want to dwell on aspects of the design that were particular to the YourWellness application, we think it is important to highlight some of these issues to other designers of such applications for older adults. While nice aesthetics are important in any application, ensuring data is easily interpreted and can be acted upon is critical. There is also balance to be attained, between designing something that is easily understood and thus requires little iterative explanation or 'help' guidance and ensuring that participants gain maximum benefit from the application. Customisation seems a potential solution, for example allowing users to 'switch off' instructions or help prompts when they are comfortable that they fully understand the application. The downside is that another level of complexity is introduced for older adults, in terms of additional functionality that they must learn.

Graph data was well understood by all participants, as were messages. "Oh the graph is good, it tells you how you're doing. I've always been a good sleeper, but if for some reason I wasn't I'd want to know why. I'd prefer to see it in the 70 s and $80 \mathrm{~s}(\%$ on the graph) than in the 30s and 40s and I'd go through the motions of getting it (the graph) back up there" (p64). "It was good to see the graph, you know and some days you were a way down and you'd say to yourself 'I've got to pick myself up a little bit here and I just have to get out'. Cos there are days you just don't feel like it. You'd sit in your pyjamas all day long and you'd say to yourself after that 'It's time I bucked myself up here. So you'd find you were moping about less. It was like a little prompter" (p69). Not only were the graphs understandable, they provided the greatest awareness of wellbeing.

\subsection{Increased Awareness of Wellbeing}

The main reason provided as to why participants found YourWellness useful is that it increases one's awareness of wellbeing. All seven participants commented that the application increased their awareness of their wellbeing, and all stated that in general, they want to be more aware: "I think a lot of people take (health) things for granted, including myself. You think you feel fine and everything's going ok. But it's no harm to see these things" (p60). The first level of awareness that arose from participant evaluations was that of simply answering the questions 
daily. It was felt that the questions that were asked were "perceptive and focused" and "they got to the heart of the matter and made you think." Participants found the graphs particularly useful for reviewing data "to see if your patterns have changed". Participants reported reflecting on those days or weeks when their scores were lower than normal and trying to remember what had happened during this time that might have caused the low score. The advice messages were overall thought to be very useful and provided an awareness of "best practice" with regard to maintaining healthy behaviours. "I think the advice, for me, that's the best part of it. There's brilliant advice there. And stuff I didn't know myself" (p66). "They're useful and they're common sense, though I wouldn't have necessarily known all that stuff"' (p59).

\subsection{Participant Behaviours}

Evaluating behaviour change is challenging and complex. As highlighted by Klasnja, Consolvo and Pratt "Behaviour change is a complex, long-term process with high relapse rates. To convincingly demonstrate that a technology contributed to such a process requires large-scale, long-term studies than can typically not be done with early-stage and error-prone systems frequently developed in HCI" [13]. Thus while we were interested in how participants might have altered their behaviours during the trial, we were not evaluating behaviour change in the traditional sense.

During interviews participants were asked whether they altered their behaviour, or tried new actions based on the feedback provided by the application. All participants said they read the advice messages regularly. However, not all followed the advice. Some felt they were already following many of them. For example, those who were particularly active felt they didn't need to do anything additional to what they were already doing. Yet they still reported finding the messages useful with one person stating that they "reinforced my confidence in what I was already doing" (p66).

Participants reported following the sleep messages more so than mood or social. This was expected, as all but two of the participants reported poor sleep hygiene. "I could see through it (the app) that I don't really sleep enough. It made me realize I was only sleeping 5-6 hours and I'd think it shouldn't really be that bad" (p59). Three participants reported actively following an advice message. Participant 69 said "some of the sleep stuff was good, like not drinking coffee or alcohol before bed. I gave them a try." He also spoke of how viewing the graph data on his mood made him "buck up" and that "you'd find you were moping about less".

Participant 64 said the questions on social interaction prompted him to be more socially active: "If you weren't talking to someone for a while, the questions would make you think of that and that you should go talk to that person. They were good prompts". Participant 65 reported changing his sleeping pattern: "I did (follow the messages). One of them especially. Not to drink tea or coffee before you go to bed. I used to drink tea about 11.30 or 12 at night before I went to bed. So I stopped that and now I sleep better. I'd have water now instead." Future work will evaluate sustained behaviour change over longer time periods.

\subsection{Maintaining Motivation}

Given that one of the primary goals of health self-management applications is to maintain a healthy state of wellbeing and prevent decline, it is important that people are motivated to use these applications for the long term. Some studies have examined older adults' acceptability of web-based health applications and found that factors such as perceived usefulness, ease of use and healthcare knowledge are important indicators of acceptance [22]. In this section we discuss how motivation and long-term adherence might be achieved by highlighting potential positive motivators as well as negative. Participants who continued to use the application past the initial 2-month trial period reported doing so because of the usefulness of the application in terms of it increasing their awareness of wellbeing and because the messages were useful for keeping an eye on your health. The questions were highlighted as the primary reason, however, for drops in level of adherence to self-reporting.

Interest/enjoyment scored lowest in terms of the motivation items assessed using the IMI. Both the types of questions being asked, and their frequency, had a negative effect on motivation. It was also felt that some of the questions were too 'clinical' and "slightly complicated the way they were worded" (p67). Participants also expressed concern that some older adults might become upset or distressed when answering some of the mood related questions. Following our workshops with clinicians, we had decided to use the Center for Epidemiological Studies Depression Scale (CES-D) [24] to assess mood, as this would provide a valid clinical measurement, which clinicians felt was desirable (if participants might want to at some point share this information with a clinician). However, we have since decided that these might not be the most feasible or the best questions to ask daily in the home. For example, one of the question items in the CES-D asks how often in the past week you have felt depressed, while another asks how often you felt your life was a failure. For someone who is feeling down, these questions might have the effect of causing further distress. We have thus decided to ask 'global' daily mood questions (e.g. 'how happy/sad/anxious/angry do you feel today) with more clinical measures such as the CES-D only being used to gain a more indepth picture if a person is continually reporting low mood.

Participants found the questions repetitive: "I found that daily you were asked the same questions. I don't think there would be that much change from day to day" (p64). We asked participants how often they felt they should answer such questions, but no general consensus emerged. The advice messages were also described as too repetitive. While the content of the messages varied, they were all in text format. Participants suggested adding videos, links to documentaries or articles they could read and these are integrated in the new design.

While participants didn't find the activity of using the application to be enjoyable, they also didn't feel it was a 'boring' activity: "Gosh no, it wasn't boring. I didn't look at it as enjoyable. It was something you did every day and you got your answers back and that" (p69). Participants also reported that it was not a 'fun' activity. This raises a question around whether health apps for older adults should be fun, or whether usefulness and competence are sufficient to maintain motivation and long term use. In other studies we conducted as part of the design of YourWellness, participants reported that they felt it important that a health related application should retain a level of seriousness and should not be game-like. Thus, we integrated a daily trivia question into the application as while participants might find this a fun activity, it was also considered a way of keeping the brain "ticking over". Future work will consider further ways to introduce hedonics aspects into wellness applications to encourage long-term usage.

High scores recorded for value/usefulness indicate that participants would like to use such an application again in the future. Some participants also highlighted other uses for the application, or mentioned other people they thought might benefit 
from it. Some of the younger participants (p59, p66) stated they would like their parents to fill in something like this, and to share the results with family members. Another (p60) commented: "I have neighbours it would be brilliant for, especially men." Another felt it would have been great for his mother when she was alive, as all his siblings were living away from her: "If she could have kept track of her mood it could have been communicated to us or her GP then we might have understood her better. We could have said 'look, we have to do something here, she's not a happy camper", (p66).

Given our findings, it is likely we can increase interest and thus motivation to use the application by personalizing it to include areas of wellbeing of interest to particular individuals, as well as through greater variation of content. Our next deployment will examine this and the impact it has on increasing adherence and motivation.

\subsection{Design Implications}

Our findings have a number of implications for the design of selfmanagement applications for older adults. Regarding YourWellness, a number of redesigns or system changes have been made. Firstly, our 'database', maintained through the management system, now has a much wider variety of question sets (i.e. not only mood, sleep and social, but physical activity, falls, diet management) and also a larger variety of questions within each of these sets. Our educational/advice feedback has also been updated to include multimedia content such as videos, interactive images and practical information (such as local classes or events). Each day a person receives one new piece of feedback, relevant to their current health state. This information is then archived into categories, such as mood, sleep etc. (see Figure 1c). The ability to add annotations to graph data to provide context was requested and has been integrated into our graph interface.

We recognize that there are limitations to our study. We had a relatively small sample size, due mainly to having a limited number of devices. However, we have since received additional funding to expand this trial. More details on how participants used the app would have been useful, such as how often they viewed the feedback wheel, graph and messages. Our new trial will examine these features. There were also technical issues during the trial that affected participants' usage of the application. However, this research has provided us with an increased understanding of older adults' attitudes and behaviours in relation to wellbeing self-management that can facilitate the creation of new, personalized health and wellbeing interventions for this population.

\section{Conclusion and Future Work}

There are a number of things we have learned from this study that we believe are applicable to designing wellness self-management applications for older adults in general. We believe this paper will provide researchers with a greater understanding of how older adults interpret wellbeing feedback over a longitudinal period and thus how best to design feedback for this population. Specifically, we believe the following points will be useful to other designers of wellness technologies for older adults to ensure such applications are accepted and adopted:

- Self-reporting increases awareness of wellbeing and is viewed as a positive thing. Much research in this area aims to reduce user involvement, through using sensors to gather data for example, to ensure minimal burden on the user. However, our research found that selfreporting (actually answering questions) supports reflection, which is important in wellness selfmanagement and which can thus potentially promote acceptance and adoption. Research by $\mathrm{Li}$ found that maintaining a diary alongside sensorised data collection of activity levels made participants more aware of their physical activity levels [17, Chapter 3]. Thus supporting active input of information from people may increase awareness beyond that which passive data collection provides. However, such reporting should not place too much burden on the person as this might affect longterm usage.

- $\quad$ Long-term adherence is likely going to be achieved by keeping the application personal and 'fresh' - i.e. ensuring questions and feedback are not repetitive, are engaging and introducing something new each day, such as an interesting quote, joke or trivia question.

- Older adults are interested in self-managing their wellness, but aspects that are important to them personally. There must be a gained benefit. Providing a 'menu' of wellness parameters to monitor may support this.

- Clinically worded questions may cause confusion or may stir up negative emotions. They may therefore be difficult to answer outside of a clinical setting. Selfreport applications for older adults should be mindful that the language used is easily understandable and does not lead to upsetting emotions.

- Designing feedback that is easily interpretable is important. In the case of YourWellness, line graphs were easily understood and the preferred method of feedback. Graphs allowed participants to 'dig deeper' into their wellbeing trends and to reflect on anomalies in the data.

- Supporting the provision of context, through user annotations for example, is important to facilitate reflection on past events that might have caused a change in reported wellbeing. The importance of context in supporting interpretation of anomalies in behaviour has been highlighted in previous studies [16].

The real benefit of wellness self-management applications such as YourWellness can only be realized if they are used long-term. While this study didn't evaluate the health benefits of the application, we did discover that it increased peoples' awareness of their wellbeing and that they found the advice/educational feedback to be useful. In terms of future work, the application will again be deployed for long-term use in a number of contexts. In future trials, we will evaluate health/wellness benefits through standard clinical questionnaires administered pre- and post- trial.

\section{ACKNOWLEDGMENTS}

The authors would like to acknowledge Enterprise Ireland who funded CASALA under the Applied Research Enhancement programme.

\section{REFERENCES}

[1] Bardram, J.E., Frost, M., Szanto, K., Faurholt-Jepsen, M., Vinberg, M. and Vedel Kessing, L. Designing mobile health technology for bipolar disorder: A field trial of the 
MONARCA system. In Proc. CHI 2013, ACM press (2013), 2627-2635.

[2] Brooke, J. SUS - A quick and dirty usability scale. In Usability Evaluation in Industry, 189, Taylor \& Francis.

[3] Consolvo, S. and Landay, J.A. Designing for behaviour change in everyday life. In IEEE Computer, 42, 6.

[4] CSO Ireland report Ageing in Ireland, 2007.

[5] Deci, E.L. and Ryan, R.M. Intrinsic motivation and selfdetermination in human behaviour. Springer, 1985.

[6] Dewsbury, G., Clarke, K., Rouncefield, M. and Sommerville, I. Depending on digital design in inclusivity: extending inclusivity. In Housing Studies, 19, (5), 2004, 811-825.

[7] Doyle, J., Bailey, C., Dromey, B. and Ni Scanaill, C. BASE - An interactive technology solution to deliver balance and strength exercises to older adults. In Pervasive Health, 2010.

[8] Doyle, J., O’Mullane, B., McGee, S. and Knapp, B. YourWellness: Designing an application to support positive emotional wellbeing in older adults. In British Computing Society HCI, 2012.

[9] Doyle, J., Skrba, Z., McDonnell, R. and Arent, B. Designing a touch screen communication device to support social interaction amongst older adults. In BCS HCI, 2010.

[10] Fogg, B.J., Eckles, D., Bogost, I. et al. Mobile Persuasion: 20 Perspectives of the Future of Mobile Behaviour Change, 2007.

[11] Gellis, Z.D., Kenaley, B., McGinty, J. et al. Outcomes of a telehealth intervention for homebound older adults with heart or chronic respiratory failure: A randomized control trial. In The Gerontologist, 134, 2012.

[12] Jimison, H., Pavel, M., Hatt, W.J., Chan, M., Larimer, N. and $\mathrm{Yu}, \mathrm{C} . \mathrm{H}$. Delivering a multi-faceted cognitive health intervention to the home. In Gerontechnology, 9, 2, 2010, 224.

[13] Klasnja, P., Consolvo, S. and Pratt, W. How to evaluate technologies for health behaviour change research. In $\mathrm{CHI}$ '11, 2011.

[14] Koch, S. Home tele-health current state and future trends. International Journal of Medical Information, 75, 2006, 565576.

[15] Lane, N.D., Mohammod, M., Lin, M. et al. BeWell: A smartphone application to monitor, model and promote wellbeing. In Pervasive Health '11, 2011.

[16] Lee, M.L. and Dey, A.K. Reflecting on pills and phone use: Supporting awareness of functional abilities for older adults. In CHI 2011, pp. 2095-2104.
[17] Li, I. Personal informatics and context: Using context to reveal factors that affect behaviour. $\mathrm{PhD}$ thesis, Carnegie Mellon University.

[18] Li, I., Dey, A.K. and Forlizzi, J. Understanding my data myself: Supporting self-reflection with ubicomp technologies. In UbiComp '11, 2011.

[19] Lin, J.J., Mamykina, L., Lindtner, S., Delajoux, G. and Strub, H.B. Fish 'n' Steps: Encouraging physical activity with an interactive computer game. In UbiComp '06, Springer, 261278.

[20] Marcu, G., Bardram, J.E. and Gabrielli, S. A framework for overcoming challenges in designing persuasive monitoring and feedback systems for mental illness. In Pervasive Health '11, 2011.

[21] McCullagh, P.J., Nugent, C.D., Zheng, H. et al. Promoting behaviour change in long term conditions using a selfmanagement platform. In Designing Inclusive Interactions, 2010, Springer, 229-238.

[22] Or, C., Karsh, B-T., Severtson, D.J. et al. Factors affecting home care patients' acceptance of a web-based interactive self-management technology. In JAMIA, 18, 2011, 51-59.

[23] NHS National Institute for Health and Care Excellence (NICE). http://www.nice.org.uk/

[24] Radloff, L.S. The CES-D scale. A self-report depression scale for research in the general population. In Applied Psychological Measurement, 1, (3), pp. 385-401, 1977.

[25] Ryan, R.M. and Deci, E.L. When rewards compete with nature: The undermining of intrinsic motivation and selfregulation. Intrinsic and Extrinsic Motivation: The Search for Optimal Motivation and Performance. San Diego: Academic Press, 2000.

[26] Thomson, H.J., Demeris, G., Rue, T. et al. A holistic approach to assess older adults' wellness using e-health technologies. In Telemedicine Journal and e-Health, 17, 10, 2011, 794-800.

[27] Turner, K. et al., Arnott, A. et al., Gray, P.D. et al. Grand challenge in assisted living - Home care technologies. Match Consortium, UKCRC Grand Challenges in Computing Research, 2010, Edinburgh.

[28] US Census Bureau, National Population Projections, 2008.

[29] Uzor, S. and Baillie, L. Exploring and designing tools to enhance falls rehabilitation in the home. In Proc CHI 2013, ACM press (2013), 1233-1242.

[30] World Health Organisation. Diabetes action now: An initiative of the world health organization and the international diabetes federation. WHO Library, 2000. 